PLANT PHYSIOLOGY

\section{WEB WATCH}

- http://mww.ebi.ac.uk Biology + information Biology plus information equals bioinformatics - the subject at the heart of the European Bioinformatics Institute (EBI) web pages. The EBI is a non-profit academic institution that specializes in research and services in bioinformatics, and has close ties with the European Molecular Biology Laboratory (EMBL). Its

mission is "to ensure that the growing body of information from molecular biology and genome research is placed in the public domain and is accessible freely to all facets of the scientific community in ways that promote scientific progress".

The EBI is organized under three 'programmes' service, research and industry, and these general themes are reflected on the web site. The home page provides links to a number of databases, ranging from standard nucleotide- and protein-sequence databases, to

sequence-structure

classification databases and sequence-mapping databases, several of which are hosted by the EBI itself.

The web site also contains links to a number of tools; again, some of these are hosted by the EBI. One example is DALI, a service for comparing protein structures in three dimensions to reveal biologically interesting similarities. Another success story is CORBA, which

allows objects and applications to be interconnected, regardless of a computer's software hardware or geographical location. Through the EBI's CORBA servers, all users can access the EMBL nucleotidesequence database. And should you wish to submit a sequence to this database (or, indeed, many of the other databases listed), the EBI site contains detailed guidelines for doing so.

Alison Mitchell pectate lyase.

\title{
Toughening up soft centres
}

Some fruits remain relatively firm as they ripen, whereas others - such as strawberries and avocados soften until they melt in the mouth. Such fruits quickly become overripe, even after picking, giving them a very short 'shelf life'. Such softening was thought to occur mainly from hydrolytic enzymes, such as polygalacturonase, breaking down the fruit's cell walls. Now, José Mercado and colleagues report in Plant Physiology that they have identified a new player in the process -

Pectate lyases have been extensively studied in pathogenic bacteria, where they aid infection of higher plants by breaking down the hemicellulose pectin in their cell walls. They have a highly regular $\beta$-helical structure, and attack pectins by a

\section{PROTEIN DYNAMICS}

\section{A motion picture}

We've had a rather static view of enzymes over the years, despite the wealth of information that has come from both classical enzymology and structural biology. However, enzymatic catalysis and internal protein dynamics are closely linked, so how do we begin to study the dynamics of catalysis, ask what internal motions occur, and decide whether

$\beta$-elimination reaction rather than the hydrolytic approach of most polysaccharide-degrading enzymes. Pectate lyases are also present in pollen, where they aid germination and growth of the pollen tube through the tissues of the stigma and style.

A pectate lyase gene had previously been isolated from ripe strawberries, so Mercado and colleagues decided to investigate its function by reducing its expression in transgenic plants using an antisense sequence of the gene under the control of a 35S promoter.

Three of the 41 independent transgenic lines they produced showed no significant decrease in the size and amount of fruit produced and yet had no detectable pectate lyase expression. However, the fruit that they produced were much

these movements are related to catalytic action?

In Science, Kern and colleagues now describe studies using NMR to characterize enzyme dynamics during substrate turnover in the human enzyme cyclophilin A (CypA). The cyclophilin family of enzymes catalyse the cis/trans isomerization of $\mathrm{X}$-proline peptide bonds, where ' $\mathrm{X}$ ' is any amino acid.

Previous methods to study enzyme dynamics during catalysis have looked at global motions or the motions at a specific molecular site, but NMR can simultaneously investigate movements at many atomic sites. Kern and co-workers therefore studied the movements of 160 amide nitrogen nuclei in the

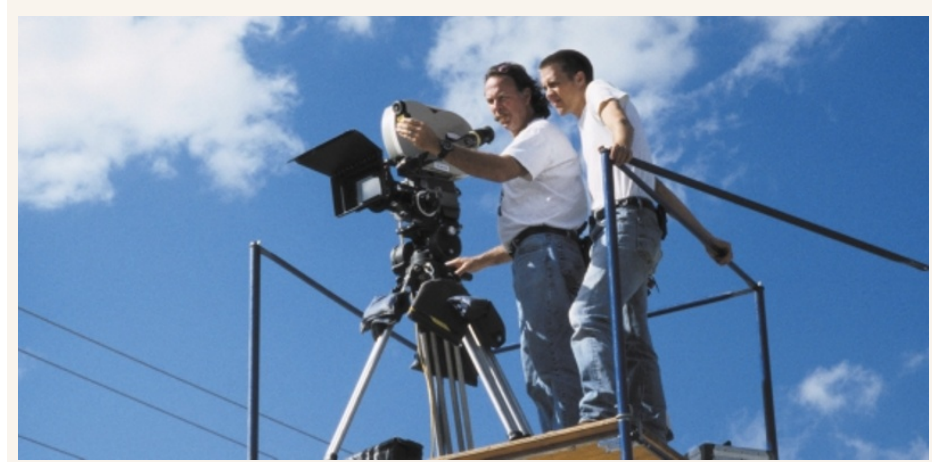

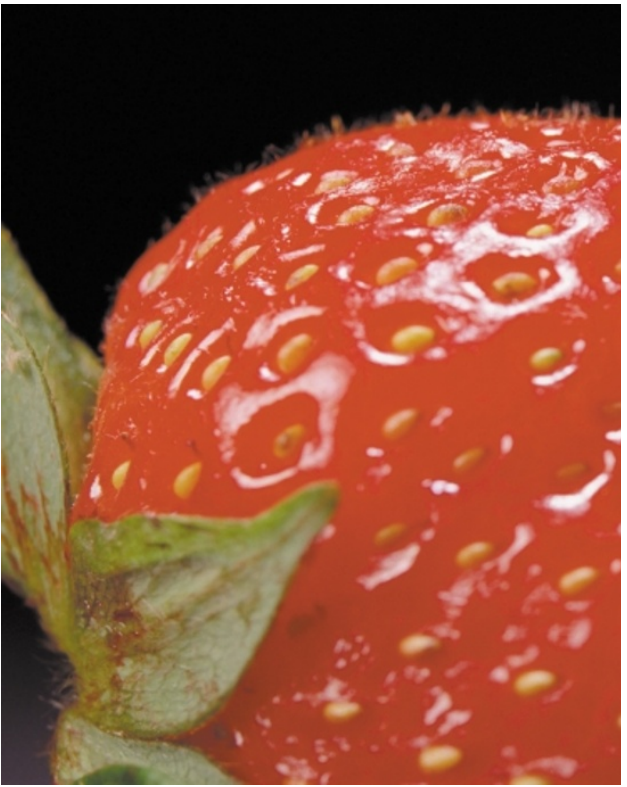

firmer than those from control plants, and far fewer had become over-ripe and 'squashy' four days after picking.

The researchers do not comment on the taste of their transgenic strawberries, although less subjective measures of fruit quality, such as the size, colour and concentration of soluble solids, were not altered. Along with improved shelf life, such transgenic plants might prove a boon to jam

protein backbone of CypA while it was actively catalysing isomerization.

Following substrate addition, the authors found that NMR

relaxation parameters that are sensitive to very fast motions (on the pico- to nanosecond time scales) did not detect any major motional changes. However, for 10 of the 160 probe nuclei, they observed changes in the $R_{2}$ relaxation parameter that is sensitive to motions on the microto millisecond time scales.

For nine of these 10 residues most of which lie in the region of the substrate binding site - Kern and colleagues found that the changes in $R_{2}$ are likely to be dominated by substrate binding and dissociation. However, the authors observed changes in $R_{2}$ for arginine 55 that indicate a role for this residue in both binding and isomerization.

$\mathrm{R} 55$ is an essential catalytic residue, the side chain of which is hydrogen bonded to the substrate. The authors observed that the motion of the amide nitrogen of $\mathrm{R} 55$ is strongly correlated with the microscopic rates of substrate 


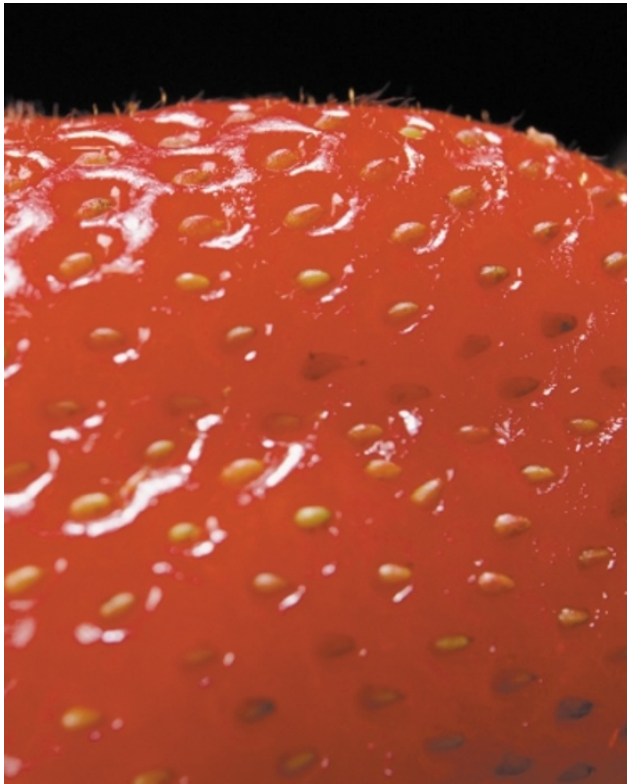

makers, who require fruit with high levels of pectin as, when boiled, this causes jam to set. This approach might also be applicable to other fruits, as a pectate lyase has already been found in ripening bananas.

Christopher Surridge, Senior Editor, Nature

(2) References and links ORIGINAL RESEARCH PAPER Jiménez-

Bermúdez, S. et al. Manipulation of strawberry fruit softening by antisense expression of a pectate lyase gene. Plant Physiol. 128, 751-789 (2002)

turnover, and concluded that this amide nitrogen is likely to be detecting motional changes that are linked to transition-state rearrangements in the protein and/or substrate. By combining their results with structural data, the authors also predicted a reaction trajectory for CypA.

Kern and co-workers have described an elegant approach for identifying dynamic 'hot spots' during catalysis, and have found that the time scales of enzyme dynamics match those of substrate turnover. Ultimately, side-chain dynamics will be required to obtain a more detailed motion picture of catalysis, but NMR relaxation measurements during catalysis promise to be invaluable for understanding both enzyme dynamics and their connection with catalysis.

Rachel Smallridge

(2) References and links ORIGINAL RESEARCH PAPER Eisenmesser, E. Z. et al. Enzyme dynamics during catalysis. Science 295, 1520-1523 (2002)

\section{WEB SITES}

Encyclopedia of Life Sciences: www.els.net NMR spectroscopy for monitoring molecular dynamics in solution

Dorothee Kern's laboratory: http://www.bio. brandeis.edu/faculty01/kern.html

\section{APOPTOSIS}

\section{Deadly FLAME}

Human transcription factor IIIC (TFIIIC) is a multi-subunit transcriptional complex that recognizes the promoters for transfer RNA and virus-associated RNA genes. According to a report in Cell Death and Differentiation, it also turns out to be an unexpected binding partner for a newly discovered death-effector domain (DED) protein - FLAME-3.

Emad Alnemri and colleagues identified FLAME-3 in a search for sequences homologous to DED-containing proteins, which are important in death-receptor signalling. FLAME-3 shows almost $50 \%$ identity to another DED-containing protein, DEDD, and both proteins are localized to the nucleus. Co-immunoprecipitation experiments showed that the two proteins can form homo- and heterodimers, and that they can both also bind to cellular FLIP (c-FLIP), a protein that antagonizes the death-receptor pathway.

The authors next did a yeast twohybrid screen for other proteins that interact with DEDD, and pulled out the TFIIIC102 subunit of TFIIIC. Coimmunoprecipitation and GST-pulldown experiments confirmed that TFIIIC102 interacts with both DEDD and FLAME-3 in vitro. Alnemri and colleagues then co-transfected cells with GFP-tagged TFIIIC102 and DEDD or FLAME-3. In both cases, the TFIIIC102 relocalized from the cytoplasm to the nucleus, hinting that DEDD and FLAME- 3 could act as chaperones to translocate TFIIIC102 across the nuclear membrane.

Finally, the authors showed that overexpression of DEDD or FLAME-3 can inhibit the transcriptional machinery, perhaps by sequestering TFIIIC102, consistent with a role for these proteins in regulating TFIIIC.

$$
\text { Alison Mitchell }
$$

(Q) References and links ORIGINAL RESEARCH PAPER Zhan, $Y$. et al. Death effector domain-containing proteins DEDD and FLAME-3 form nuclear complexes with the TFIIC102 nuclear complexes with the THIC102 Cell Death Diff. 9, 439-447 (2002)

\section{IN BRIEF}

\section{ADHESION}

Integrins regulate GTP-Rac localized effector interactions through dissociation of Rho-GDI.

Del Pozo, M. A. et al. Nature Cell Biol. 4, 232-239 (2002)

Integrin-mediated cell adhesion is required to translocate the small GTPase Rac to the plasma membrane, where it can interact with its effectors. In this study, the authors used fluorescence resonance energy transfer to show that Rac only interacts with its effectors at specific regions at the edges of cells. This was due to the ability of integrins to target Rac to these regions and to dissociate it from Rho-GDI, which binds to cytoplasmic Rac and blocks effector binding.

\section{SUMOYLATION}

Members of the PIAS family act as SUMO ligases for cJun and p53 and repress p53 activity.

Schmidt, D. \& Müller, S. Proc. Natl Acad. Sci. USA 99, 2871-2877 (2002)

Like ubiquitylation, sumoylation requires an E1-activating enzyme and an E2-type conjugating enzyme, Ubc9. Until now, Ubc9 was also thought to be sufficient for substrate recognition, which, in the case of ubiquitylation, is carried out by $\mathrm{E} 3$ ligases. Now, however, Schmidt and Müller report that protein inhibitor of activated STAT (PIAS) proteins can function as specific SUMO ligases, in a similar manner to E3 ubiquitin ligases, and can mediate the sumoylation of p53 and c-Jun. PIAS-mediated sumoylation of p 53 markedly repressed $\mathrm{p} 53$ 's transcriptional activity, indicating a role for the PIAS-SUMO pathway in transcriptional regulation.

\section{CELL SIGNALLING}

Cbl-CIN85-endophilin complex mediates ligandinduced downregulation of EGF receptors.

Soubeyran, P. et al. Nature 416, 183-187 (2002)

The endophilin-CIN85-Cbl complex mediates liganddependent downregulation of c-Met.

Petrelli, A. et al. Nature 416, 187-190 (2002)

The adaptor protein $\mathrm{Cbl}$ is involved in downregulating receptortyrosine kinases in response to ligand-induced activation by binding, through its Src-homology-2 (SH2) domain, to phosphorylated tyrosine residues. It then targets the receptors for ubiquitylation and subsequent degradation in lysosomes. But two new studies in Nature now show that $\mathrm{Cbl}$ also has a regulatory role in receptor internalization that is functionally separable from its ubiquitylation activity. Both research groups found that $\mathrm{Cbl}$ interacts, through its carboxyl terminus, with a complex that consists of endophilins - components of clathrin-coated vesicles that mediate endocytosis - and Cbl-interacting protein of 85 $\mathrm{kDa}, \mathrm{CIN} 85$. The model proposed is that endophilin can alter the shape of the plasma membrane, thereby promoting membrane invagination, and that CIN85, an adaptor protein, might regulate receptor transport. 